\title{
TRIANGULACIÓN DE MÉTODOS E INSTRUMENTOS DE EVALUACIÓN EN EDUCACIÓN SUPERIOR
}

\author{
Triangulation of methods and assessment tools in Higher Education \\ Triangulação de métodos e instrumentos de avaliação no Ensino Superior
}

\section{Aroa Cintas Gómez (1)}

(1) Escuela Universitaria de Turismo Ostelea - Universitat de Lleida, Barcelona, España. Teléfono: +34 663656106. Correo electrónico: aroacintasgomez@gmail.com

\section{Resumen}

En términos pedagógicos, los planteamientos multivariados en cuanto a los instrumentos, técnicas y métodos de recogida de información son claves para poder tener una perspectiva holística del proceso de enseñanza, aprendizaje del estudiantado y la comprobación de la adquisición de competencias requeridas. Desde un paradigma tradicional, la evaluación del aprendizaje se constituye por la evaluación de los conocimientos adquiridos y de una forma unidireccional del profesor hacia el estudiante. Sin embargo, el proceso de convergencia EU lleva consigo una serie de cambios en la misión de la universidad en cuanto al proceso de enseñanza y aprendizaje de los estudiantes. La educación superior está enfocada a nuevo planteamiento pedagógico donde el alumno ya no es un stakeholder pasivo, sino activo en su propio proceso de enseñanza y aprendizaje. La siguiente comunicación, presentará el plan evaluativo configurado por múltiples metodologías de evaluación e instrumentos conforme a las competencias requeridas del Proyecto Transversal que se realizó en el Grado oficial en Ostelea.

Palabras clave: Evaluación; calidad educativa; métodos, rúbrica, proceso enseñanzaaprendizaje

\begin{abstract}
In pedagogical terms, the multivariate approaches in terms of instruments, techniques and methods of collecting information are key to being able to have a holistic perspective of teaching and learning process of the student. From a traditional paradigm, the evaluation of learning is constituted by the evaluation of the acquired knowledge and in a unidirectional way from the teacher to the student. However, the process of
\end{abstract}


European convergence brings with it a series of changes in the mission of the university in terms of the teaching and learning process of students in higher education, higher education is focused on a new pedagogical approach where the student is no longer a passive stakeholder, but active in their own teaching and learning process. The following communication will present the evaluative plan configured by multiple evaluation methodologies according to the required competences of the Project Transversal that was made in the official Grade in Ostelea

Keywords: assessment; educational quality; methods, rubric, teaching-learning process.

\section{Resumo}

Em termos pedagógicos, as abordagens multivariadas em termos de instrumentos, técnicas e métodos de recolha de informação são aspetos fundamentais para ter uma perspectiva holística do processo de ensino, aprendizagem do aluno e verificação da aquisição das competências esperadas. Tendo em conta, oparadigma tradicional, a avaliação da aprendizagem constitui-se pela avaliação do conhecimento adquirido e de uma forma unidirecional do professor para o aluno. No entanto, o processo de convergência EU traz consigo uma série de mudanças na missão da universidade em termos do processo de ensino e aprendizagem dos alunos. $\mathrm{O}$ ensino superior está focado numa nova abordagem pedagógica, onde o aluno não é mais um stakeholder passivo, mas sim, ativo em seu próprio processo de ensino e aprendizagem. Esta comunicação apresentará o plano de avaliação configurado por múltiplas metodologias de avaliação e instrumentos, de acordo com as competências requeridas pelo Projeto Transversal que se realizou na Licenciatura oficial em Ostelea.

Palavras-chave: Avaliação; qualidade educativa; métodos, rubrica, processoensinoaprendizagem

\section{Introducción}

El termino triangulación de instrumentos, en una primera instancia estaba contextualizado en el ámbito de la investigación como procedimiento de control implementado para garantizar la confiabilidad entre los resultados de cualquier investigación (Betrián, Galite, Merino, Monclús \& Macarulla, 2013). Asimismo, la misma conceptualización ha sido trasladada en todos los niveles educativos. En el caso 
de la Educación Superior, el proceso de convergencia europea lleva consigo una serie de cambios en la misión de la universidad en cuanto al proceso de enseñanza y aprendizaje de los estudiantes, entendido como un todo en el que los profesores y alumnos compartan la responsabilidad. Un nuevo planteamiento pedagógico donde el alumno participa activamente en todo el proceso de enseñanza y aprendizaje, incluida la evaluación (Martínez, 2008) que debería, según L. Noguero (citado en Martínez, 2008), afrontarse como un elemento educativo más, generador de la participación, a través de la cual, tanto el alumno como el docente pueden reflexionar y realizar propuestas de mejora de los procesos que vayan teniendo lugar, desterrando el carácter coercitivo que, tradicionalmente, ha acompañado a la evaluación.

En cualquier caso, la valoración del grado de adquisición de competencias no debe ser unidireccional del profesor hacia el estudiante. Tal y como señala (Martinez, 2007) con la creación del Espacio Europeo de Educación Superior (EEES), se produce un cambio en el papel del profesor pues de ser el protagonista de la enseñanza al ser la persona que estructura el proceso de aprendizaje, el supervisor y director de trabajos, pasa a ser "un acompañante en el proceso de aprender, que ayuda al que estudia a alcanzar ciertas competencias" (González \& Wagenaar, 2003).

Para poder tener un resultado preciso del resultado de aprendizaje del alumno, hace falta tener diferentes fuentes de información donde se evidencie el aprendizaje adquirido y obtener así una evaluación holística.

En cuanto a qué técnicas, modelos o instrumentos son más idóneas para elaborar un plan evaluativo, lo cierto es que no existe unanimidad a la hora de clasificar los diferentes instrumentos de medida que suelen utilizarse en el proceso de recogida de datos en una evaluación (Ruiz, 2004) Asimismo, cada método tiene sus características que pueden ser más afines o no a los objetivos que previamente se plantean.

En el caso de los métodos utilizados en el Proyecto Transversal; están compuestos por la evaluación por parte del profesorado, la evaluación cooperativa y la co-evaluación. Dichos métodos se sustentan en rúbricas y cuestionarios mixtos con preguntas cerradas y abiertas.

\section{Contextualización}

En el presente, se detalla el plan evaluativo que se ha desarrollado en el Proyecto Transversal del Grado Oficial en Turismo y Ocio. Dicho proyecto es común a todas las 
asignaturas de primer curso del Grado, y tiene como objetivo aplicar los conocimientos de una manera integral; presenta el análisis de un proyecto o empresa turística a partir de un documento final que incorpora el análisis de diversos aspectos que han sido trabajados en todas las asignaturas de primer y segundo curso. El profesor de cada asignatura establece el contenido y el sistema de evaluación del apartado que conforma el proyecto transversal; sin embargo, para todas las asignaturas el proyecto transversal representa un $10 \%$ del conjunto de actividades de evaluación de la asignatura y se evalúa mediante la triangulación de métodos e instrumentos evaluativos descritos anteriormente.

\section{Diseño y desarrollo}

Respecto al diseño y desarrollo, el plan evaluativo ha sido confeccionado mediante la consecución de las siguientes fases (Tabla 1):

Tabla 1.

Fases del diseño y desarrollo del plan evaluativo. Elaboración propia (2019).

\begin{tabular}{|c|c|c|}
\hline Fase y descripción & Acción & Resultado \\
\hline $\begin{array}{l}\text { Fase } 1 . \text { Diagnóstico de } \\
\text { necesidades. }\end{array}$ & $\begin{array}{l}\text { Detección de las necesidades a través de } \\
\text { las sugerencias de los estudiantes, en este } \\
\text { caso, de la necesidad de introducir nuevos } \\
\text { métodos evaluativos para suplir sus } \\
\text { necesidades. }\end{array}$ & $\begin{array}{l}\text { Valoración de la viabilidad } \\
\text { de la detección de } \\
\text { necesidades, propuesta de } \\
\text { creación de un nuevo plan } \\
\text { evaluativo. }\end{array}$ \\
\hline $\begin{array}{l}\text { Fase 2. Establecer } \\
\text { Objetivos. }\end{array}$ & $\begin{array}{l}\text { Comprobación de las competencias y } \\
\text { objetivos del Proyecto Transversal. }\end{array}$ & $\begin{array}{l}\text { - Establecimiento de los } \\
\text { criterios e indicadores de } \\
\text { cada competencia a lograr. } \\
\text { - Ponderación aplicada a } \\
\text { cada una de las } \\
\text { metodologías. }\end{array}$ \\
\hline $\begin{array}{l}\text { Fase } 3 . \text { Selección de } \\
\text { métodos y descripciones } \\
\text { de los mismos. }\end{array}$ & $\begin{array}{l}\text { Estudio de los métodos más idóneos para } \\
\text { responder a los criterios e indicadores de } \\
\text { cada competencia. }\end{array}$ & $\begin{array}{l}\text { Decisión de utilizar: (1) } \\
\text { Evaluación colaborativa. (2) } \\
\text { Coevaluación. (3) } \\
\text { Evaluación por parte del } \\
\text { profesor. }\end{array}$ \\
\hline $\begin{array}{l}\text { Fase 4. Selección de } \\
\text { instrumentos de recogida } \\
\text { de información y } \\
\text { confecciones de estos. }\end{array}$ & $\begin{array}{l}\text { Una vez seleccionados los métodos } \\
\text { evaluativos se comprueba los } \\
\text { instrumentos más afines a cada método. }\end{array}$ & $\begin{array}{l}\text { Decisión de utilizar: (1) } \\
\text { Rúbricas. (2) Cuestionarios } \\
\text { mixtos. }\end{array}$ \\
\hline $\begin{array}{l}\text { Fase 5. Proceso de } \\
\text { comunicación del nuevo } \\
\text { plan evaluativo. }\end{array}$ & $\begin{array}{l}\text { Planteamiento de formación al claustro } \\
\text { para la utilización de estos. }\end{array}$ & Formación a los docentes. \\
\hline Fase 6. Implementación. & Ejecución del plan evaluativo. & Evaluación. \\
\hline $\begin{array}{l}\text { Fase } 7 . \text { Resultado y } \\
\text { conclusión. }\end{array}$ & Ponderación de los resultados. & $\begin{array}{l}\text { Se obtienen los resultados y } \\
\text { se realizan las propias } \\
\text { conclusiones. }\end{array}$ \\
\hline
\end{tabular}




\section{Evaluación y conclusiones}

La evaluación de la experiencia se realizó mediante la retroalimentación de los docentes implicados, satisfacción de alumnos/as y el departamento académico.

Como conclusión general, las actividades formativas de tipo cooperativo tienen un protagonismo destacado en el marco de los nuevos grados universitarios del EEES. Por ello, la evaluación del proceso de enseñanza-aprendizaje debe tener en cuenta estas metodologías y promoverlas en las diferentes actividades que se realicen dentro de un programa formativo. Las conclusiones generalmente fueron óptimas por los siguientes beneficios: evaluación más objetiva y contrastada con múltiples fuentes, desarrollo de competencias transversales (autonomía, liderazgo, autocrítica) y aumento de la motivación y autoestima (Domingo, 2008).

Asimismo, también hemos encontrado algunas resistencias ante la incorporación de los métodos e instrumentos que anteriormente he mencionado: la subjetividad por parte del alumno que no asume satisfactoriamente la responsabilidad de ser objetivo y rechazo por parte de otros alumnos por ser evaluados por sus compañeros/as. En el caso de los docentes, encontramos resistencias de cambio ante el desafío de aplicar en sus prácticas educativas, nuevos conceptos y metodologías a los utilizados anteriormente.

\section{Referencias}

Betrián, B., Galite, N., Merino, N., Monclús, G., \& Macarulla, M. (2013). La triangulación múltiple como estrategia metodológica. Revista Iberoamericana sobre Calidad, Eficacia y Cambio en Educación, 11(4), 5-24.

González, J. \& Wagenaar, R. (2003). Tuning Educational Structures in Europe. Informe final. Proyecto Piloto-Fase 1. Bilbao: Universidad de Deusto.

López, F. (2005). Metodología participativa en la Enseñanza Universitaria. Madrid: Narcea Ediciones.

Martínez, C. (2008). Una propuesta de evaluación en el EEES: el uso del portfolio en una clase de idiomas. Porta Linguarum. Revista Internacional de Didáctica de las Lenguas Extranjeras, 9, 61-74.

Martínez, M. (2007). El nuevo papel del profesor universitario en el proceso de convergencia europeo y su relación con la interacción, la tutoría y el aprendizaje autónomo. Porta Linguarum. Revista Internacional de Didáctica de las Lenguas Extranjeras, 7, 31-43. 
Ruiz, C. (2004). Evaluación de la formación. CIFO. Barcelona: Universidad Autónoma de Barcelona.

Domingo, J. (2008). El aprendizaje cooperativo. Cuadernos de trabajo social. Revista innovación y experiencias digitales educativas, 21, 231-246. 